\title{
Texture Image Segmentation Using Without Re-initialization Geodesic Active Contour Model
}

\author{
Kaibin Wang Bianzhang Yu \\ Department of Electronic and Information Engineering, Northwestern Polytechnical University, Xi'an 710072, \\ Shaanxi P.R.China
}

\begin{abstract}
A novel method of texture image segmentation is proposed, which has three advantages compared to other active contours. First, by combining the gray levels of pixels with texture information of an image, this method can be used for segmentation of a texture image or a non-texture image. Second, the method has low computation complexity because local binary pattern (LBP) is employed to extract texture features. And last, the proposed algorithm can avoid the additional computation problem without reinitialization of signal distance function repeatedly. The segmentation tests show that the proposed segmentation method is efficient, accurate, fast and robust.
\end{abstract}

Keywords: Texture image segmentation, Local binary pattern, Geodesic active contour, Level set

\section{Introduction}

Texture segmentation has long been an important topic in image processing. It aims at segmenting a textured image into several regions with same texture features within one region. An effective texture segmentation algorithm is of importance in diverse areas like remote sensing, medical image analysis and image retrieval. A wide variety of segmentation methods for textured image have been proposed. These methods can fall into four categories: region growth, statistical histogram, edge detection and partial differential equations (PDE)-based algorithms [1], [2] .

Recently, PDE-based active contours models (or curve evolution) have attracted more interests for image segmentation techniques because they do not require additional edge-linking operators to establish the connectivity of edge segments. Curve evolution means to evolve deformable contours to conform to object boundaries for a given image. It can be classified as parametric active contours and geometric active contours. Parametric active contours represent curves or surfaces explicitly in parametric forms, and can lead to fast real-time implementation. But it has the difficulty in adapting the model topology during deformation. On the other hand, geometric active contours are based on level set method which represents curve or surfaces implicitly, and can handle topological changes naturally [3]. But geometric active contours have two drawbacks. One is that the computational expense is high, another is that a textured image can not be segmented properly because they do not use texture features to evolve curves or surfaces during image processing. State-of-the-art research in active contour models has concentrated on the use of texture as a guiding tool for contour evolution, and several models based on active contours for textured image segmentation have been proposed [4]-[7]. Most of these approaches involves in Gabor and wavelet, statistical and filtering or cooccurrence matrices approaches to contour evolution. The local binary pattern (LBP) operator offers an alternative approach for texture representation. It can provide more fine-grained information. In addition, the LBP texture features are invariant to any monotonic change in gray level intensities, resulting in a more robust representation of textures under varying illumination conditions. Comparative studies have show that excellent discrimination can be obtained with LBP operator and nonparametric statistical discrimination of sample and prototype distributions [8], [9].

In this paper we propose a novel algorithm for unsupervised segmentation that incorporates an LBP/Contrast or Contour-based representation of textures under a geometric active contour framework.

The organization of this paper is as follows. In section 2, we give a brief review of LBP/C features. Our model is formulated in section 3 . In section 4 , the results of its application on two textured images are given. Finally we conclude the paper in section 5.

\section{Texture description}

The texture contents of an image region are characterized by the joint distribution of local binary 
pattern and contrast features [10]. Considering neighborhoods of $3 \times 3$ pixels (Fig.1a), the LBP and C values are computed as follows: i) the original $3 \times 3$ neighborhood pixels are thresholded by the value of the center pixel (Fig.1b). ii) the values of the pixels in the thresholded neighborhood are multiplied by some binomial weights (Fig.1c) assigned to the corresponding pixels, iii) the values of the eight pixels (Fig.1d) are summed for the LBP number (169) of this texture unit, iv) contrast measure $\mathrm{C}$ is the difference between the average gray-level of those pixel which have value 1 and those which have value 0 (Fig.1b). The LBP/C distribution is approximated by a discrete two-dimensional histogram of size $256 \times b$, where b is the number of bins for $\mathrm{C}$. In order to reduce the size of histogram, we extend the uniform LBP to LBP/C operator, which can reduce the size to $\mathbf{5 8} \times$ b.

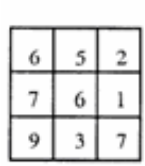

(a)

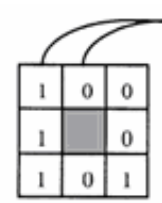

(b)

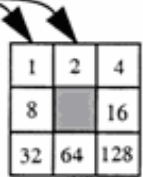

(c)

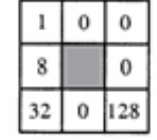

(d)
LBP $=1+8+32+128=169$

$\mathrm{C}=(6+7+9+7) / 4-(5+2+1+3) / 4=\mathbf{4 . 5}$

Fig.1: Illustration of LBP/C algorithm for texture description.

\section{LBP-based geometric active contour model}

In level set formulation of moving fronts, the fronts, denoted by $C$, are represented implicitly by the zero level set $C(t)=\{(x, y) \mid \phi(x, y, t)=0\}$ of a level set function $\phi(x, y, t)$. The evolution equation of level set function $\phi$ can be written in the following general form:

$$
\frac{\partial \phi}{\partial t}+F|\nabla \phi|=0
$$

where $F$ is the speed of evolving the curve in normal direction. A particular case is the motion by mean curvature, If $F=\operatorname{div}(\nabla \phi(x, y) /|\nabla \phi(x, y)|)$ is the curvature of the level-curve of $\phi$ passing point $(x, y)$, then a geometric active model based on the mean curvature motion is given by the following evolution equation:

$$
\left\{\begin{array}{l}
\frac{\partial \phi}{\partial t}=g\left(\left|\nabla u_{0}\right|\right) \mid \nabla \phi\left(\operatorname{div}\left(\frac{\nabla \phi}{\mid \nabla \phi}\right)+v\right), t \in(0, \infty) \\
\phi(0, x, y)=\phi_{0}(x, y), x \in \square^{2}
\end{array}\right.
$$

where $v$ is a constant, and $g\left(\left|\nabla u_{0}\right|\right)$ is an edgefunction depending on the gradient of the image $u_{0}$. In this paper, $g\left(\left|\nabla u_{0}\right|\right)$ is defined as

$$
g\left(\left|\nabla u_{0}(x, y)\right|\right)=\frac{1}{1+\left|\nabla G_{\sigma}(x, y) * I(x, y)\right|^{2}}
$$

where $G_{\sigma}$ is the Gaussian kernel with standard deviation $\sigma$.

The above stated geometric active contour model only uses the gray information of pixels to evolve curves or surfaces during image processing, so a textured image can not be segmented properly. It is necessary to introduce texture features to geometric active contour model for textured image segmentation. But the LBP/C code on a pixel can not describe its texture information completely, since texture is often defined on a local patch. We place a window around a pixel, and the feature of a pixel is representative of the texture distribution and thus the smaller misclassification. A region can also be modeled by a $\mathrm{LBP} / \mathrm{C}$ histogram [11]. The $\mathrm{G}$ statistics is used as a pseudo-metric for comparing LBP/C distributions [10]. We measured the similarity of two histograms with a two way test of interaction or heterogeneity:

$$
\begin{aligned}
G= & 2\left[\sum_{s, m}^{n} \sum_{i=1}^{n} \log f_{i}\right]-\left[\sum_{s, m}\left(\sum_{i=1}^{n} f_{i}\right) \log \left(\sum_{i=1}^{n} f_{i}\right)\right] \\
& -\left[\sum_{i=1}^{n}\left(\sum_{s, m} f_{i}\right) \log \left(\sum_{s, m} f_{i}\right)\right] \\
& +\left[\left(\sum_{s, m} \sum_{i=1}^{n} f_{i}\right)\right] \log \left(\sum_{s, m} \sum_{i=1}^{n} f_{i}\right)
\end{aligned}
$$

where $s, m$ are two sample histograms respectively, $n$ is the number of bins and $f_{i}$ is the frequency at bin $i$. The more similar the histograms $s$ and $m$ are, the smaller is the value of G. Assume edgefunction $g\left(\left|\nabla u_{0}\right|\right)=G$, and the geometric active model for textured image segmentation can be written as:

$\left\{\begin{array}{l}\frac{\partial \phi}{\partial t}=G|\nabla \phi|\left(\operatorname{div}\left(\frac{\nabla \phi}{|\nabla \phi|}\right)+v\right), t \in(0, \infty) \\ \phi(0, x, y)=\phi_{0}(x, y), x \in \square^{2}\end{array}\right.$

This model can be applied to segment a textured image. But for an image without rich texture information, the segmentation results are not 
satisfactory, since it only employs texture features to segment an image. So, we improve equation (5) further and obtain

$$
\left\{\begin{array}{l}
\frac{\partial \phi}{\partial t}=\left[\lambda_{1} G+\lambda_{2} g\left(\nabla u_{0}\right)\right] \mid \nabla \phi\left(\operatorname{div}\left(\frac{\nabla \phi}{\mid \nabla \phi}\right)+v\right), t \in(0, \infty) \\
\phi(0, x, y)=\phi_{0}(x, y), x \in \square^{2}
\end{array}\right.
$$

where $\lambda_{1}$ and $\lambda_{2}$ are non-negative constants. From equation (6), we can see that the segmentation model uses texture information of the pixels and gradient information to evolve a curve of surface. Therefore, it can be used to segment an image with texture or without texture.

If we solve equation ( 6 ) directly, the computation burden is high and curve convergence speed is slow. This is because level set function $\phi(t, x, y)$ can develop shocks, very sharp or flat shape during the evolution, which makes further computation highly inaccurate. In order to avoid these problems, a common numerical scheme is to initialize the function $\phi(t, x, y)$ and a signed distance function before the evolution, and re-initialize the function $\phi(t, x, y)$ to be a signed distance function periodically during the evolution. The standard reinitialization method is to solve the following reinitialization equation:

$$
\frac{\partial \phi}{\partial t}=\operatorname{sign}\left(\phi_{0}\right)(1-|\nabla \phi|)
$$

This re-initialization $\phi(t, x, y)$ to be a signed distance function results in additional computation. In order to accelerate segmentation for a textured image, we follow [12] to add a regularizing term $P(\phi)=\int_{\Omega} \frac{1}{2}(|\nabla \phi|-1)^{2} d x d y \quad$ in equation (6) . After formulation, the LBP-based geometric active contour model is obtained:

$$
\begin{aligned}
\frac{\partial \phi}{\partial t}= & \mu\left[\Delta \phi-\operatorname{div}\left(\frac{\nabla \phi}{|\nabla \phi|}\right)\right] \\
& +\lambda \delta(\phi) \operatorname{div}\left(\left(\lambda_{1} G+\lambda_{2} g(\nabla u)\right) \frac{\nabla \phi}{|\nabla \phi|}\right) \\
& +v\left(\lambda_{1} G+\lambda_{2} g(\nabla u)\right) \delta(\phi)
\end{aligned}
$$

The LBP-based geometric active contour model can use much larger time step to speed up the curve evolution, while maintaining stable evolution of the level set function. Moreover, it no longer requires the level set function to be initialized as a signed distance function. So the segmentation speed is increased. For all the experiments in this paper, the Dirac function $\delta(x)$ is defined by: $\delta_{\varepsilon}(x)= \begin{cases}0, & |x|>\varepsilon \\ \frac{1}{2 \varepsilon}\left[1+\cos \left(\frac{\pi x}{\varepsilon}\right)\right], & |x| \leq \varepsilon\end{cases}$

where $\varepsilon=1.5$. The upwind scheme is no longer needed due to introducing the diffusion term in equation ( 6 ) . Instead, all the spatial partial derivatives $\frac{\partial \phi}{\partial x}, \frac{\partial \phi}{\partial y}$ are approximated by the central difference. And the temporal partial derivative $\frac{\partial \phi}{\partial t}$ is approximated by the forward difference [11]. The approximation of equation (8) by the above difference scheme can be simply written as:

$$
\frac{\phi_{i, j}^{k+1}-\phi_{i, j}^{k}}{t}=L\left(\phi_{i, j}^{k}\right)
$$

where $L\left(\phi_{i, j}^{k}\right)$ is the approximation of the right hand side in (8) by the above spatial difference. So the difference equation (10) can be expressed as the following iteration:

$$
\phi_{i, j}^{k+1}=\phi_{i, j}^{k}+t L\left(\phi_{i, j}^{k}\right)
$$

\section{Experimental results and discussions}

To demonstrate the performance of the proposed method, both synthetic and natural image are used. The first test image (Fig.2(a)) is composed of two textures, and the second is of a zebra image(Fig.3(a)). The LBP/C operator constants were generally chosen as follows: A $3 \times 3$-pixel neighborhood was considered for the calculation of the $\mathrm{LBP} / \mathrm{C}$ values, whereas the window size was set to $16 \times 16$.The segmentation results are shown in Fig 2 and Fig 3, and Table 1 and Table 2 shows the parameters used in the experiments.

\begin{tabular}{|c|c|c|c|c|c|c|}
\hline & \multicolumn{6}{|c|}{ the proposed method } \\
\hline & $\mu$ & $\lambda$ & $v$ & $t$ & $\lambda_{1}$ & $\lambda_{2}$ \\
\hline Fig.2 & 0.03 & 6.0 & 3.0 & 7.0 & 1 & 0 \\
\hline Fig.3 & 0.04 & 5.0 & 2.0 & 6.0 & 0.95 & 0.05 \\
\hline
\end{tabular}

Table. 1: Parameters of segmentation image.

\begin{tabular}{|c|c|c|c|}
\hline & \multicolumn{3}{|c|}{ the method in [7] } \\
\hline & $\lambda_{i}$ & $\gamma_{i}$ & $\mu$ \\
\hline Fig.2 & 1 & 0.3 & 19500 \\
\hline Fig.3 & 1 & 0.3 & 38000 \\
\hline
\end{tabular}

Table. 2: Parameters of segmentation image. 
We contrast the segmentation accuracy and speed of the method in [4], the method in [7] and the method proposed in this paper. The error segmentation ration for the synthetic textured image is $3.28 \%$ by the method in [4], $4.54 \%$ by the method in [8], whereas $1.15 \%$ by the proposed method. From Fig 2 and Fig 3, it can be seen that our method is most accurate and fastest among the three algorithms. This is because LBP/C operator has an excellent discrimination with a low complexity, and our algorithm does not require the level set function to be re-initialized as a signed function.

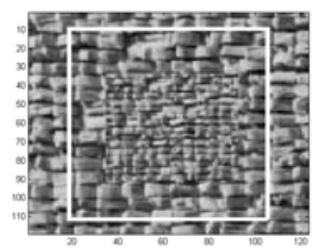

(a)

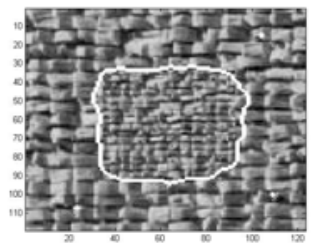

(c)

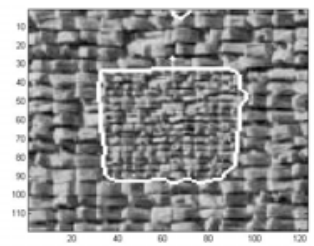

(b)

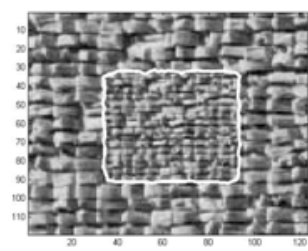

(d)
Fig. 2: Segmentation of synthetic textured image. (a)Origin image, (b) segmented image by the method in [4] (iterations=1050), (c) segmented image by the method in [7] (iterations=1100), (d) segmented image by the proposed method in this paper (iterations=200).

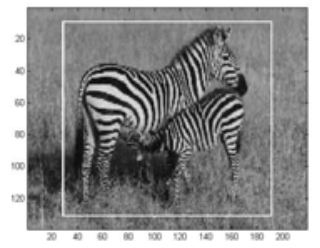

(a)

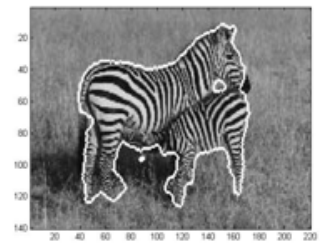

(c)

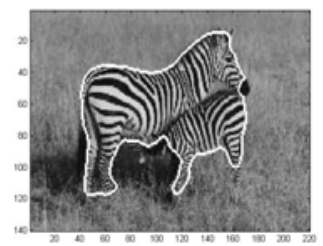

(b)

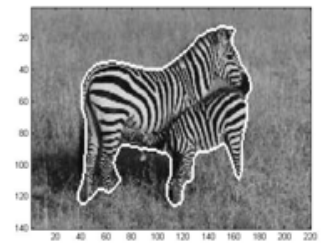

(d)
Fig.3: Segmentation of natural textured image. (a)Origin image, (b) segmented image by the method in [4] (iterations=1920), (c) segmented image by the method in [7] ( iterations=2000), (d) segmented image by the proposed method in this paper (iterations $=450$ ).

\section{Conclusions}

In this paper, we present a novel method for unsupervised textured image segmentation which embodies the advantageous of LBP/C operator and geodesic active contour. The LBP-based geometric active contour model does not require the level set function to be initialized as a signed distance function and can use significantly larger time step to speed up the curve evolution. The simulation experiments show that the proposed algorithm is accurate and efficient for various segmentation tasks.

\section{Acknowledgement}

The authors would like to acknowledge the support of NWPU seed funds (Grant No.Z200538) and national defense funds (Grant No.A3220061163). And this paper uses MATLAB codes developed by Chunming $\mathrm{Li}$, which helps author to learn without re-initialization level set model.

\section{References}

[1] T.R. Reed, J.M.H. Du Buf, A review of recent texture segmentation, feature extraction techniques. CVGIP Image Understanding, 57: 359-372, 1993.

[2] Randen and J. Husoy, Filtering for texture classification: a comparative study. IEEE Trans on Pattern Analysis and Machine Intelligence, 21(4): 291-310, 1999.

[3] C.Y. Xiao, S. Zhang, Y.Z. Chen. Fast Image Segmentation Based on a Two-Stage Geometrical Active Contour. Journal of Shanghai University, 9(1): 40-45, 2005.

[4] S.G. Chen, N. A Sochen, Yehoshua Y. Zeevi. Geodesic Active Contours Applied to Texture Feature Space. Scale-Space, Vancouver Canada, pp. 344-352, 2001.

[5] T. Lehmann, Klaus Spitzer, Texture-Adaptive Active Contour Models. Brazil, March 11-14, Proceedings, pp. 388-396, 2001.

[6] I. Karoui, R.Fabler, J.M.Boucher, J.M.Augustin, Region-Based Image Segmentation Using Texture Statistics and Level-Set Methods. ICASSP, pp. 693-696, 2006.

[7] B. Sandberg, T. Chan, L.Vese, A level set and gabor based active contour algorithm for segmenting textured images. UCLA CAM Report 02 - 39, 2002.

[8] T. Ojala, M. Pietikäinen and D. Harwood. A comparative study of texture measures with 
classification based on feature distributions. Pattern Recognition, 29: 51-59, 1996.

[9] T. Maenpaa, The Local Binary Pattern Approach to Texture Analysis- Extensions and Applications, Oulou University Press, Finland, 2003.

[10] T. Ojala, M. Pietikäinen, Unsupervised texture segmentation using feature distribution. Pattern Recognition, 32: 477-486, 1999.

[11] Q. Xu, J. Yang, S.Y. Ding, Texture Segmentation using LBP embedded Region Competition. Electronic Letters on Computer Vision and Image Analysis, 5(1): 41-47, 2004.

[12] C.M. Li, C.Y. Xu, C.F. Gui. Level Set Evolution without Re-initialization: A New Variational Formulation. In Proc. IEEE: Computer Vision and Pattern Recognition (CVPR'05), 2005. 